\title{
Special issue on deep learning-based neural information processing for big data analytics
}

\author{
Chuanchao Huang ${ }^{1}$
}

Received: 25 October 2019/Accepted: 29 October 2019/Published online: 4 November 2019

(c) Springer-Verlag London Ltd., part of Springer Nature 2019

Living in the era of big data, we have been witnessing the dramatic growth of heterogeneous data, which consists of a complex set of cross-media content, such as text, images, videos, audio, graphics, and time series sequences. Such hybrid data comes from multiple sources and hence embodies different feature spaces. This situation is creating new challenges for the design of effective algorithms and developing generalized frameworks to meet heterogeneous computing requirements. Meanwhile, deep learning is revolutionizing diverse key application areas, such as speech recognition, object detection, image classification, and machine translation, with its data-driven representation learning. Thus, it has become critical to explore advanced deep learning techniques for heterogeneous big data analytics, including data acquisition, feature representation, time series analysis, knowledge understanding, and semantic modeling. With the resurgence of deep learning architectures and learning methods, neural information processing has been applied to a variety of disciplines and proved highly successful in a vast class of applications. For instance, in the pattern recognition field, deep neural networks achieved human-like performance in recognizing, labeling and sorting images, e.g., on the ImageNet benchmark. Moreover, DeepMind's AlphaGo Zero, trained by self-play reinforcement learning, achieved superhuman performance in the game of Go. On the hardware architecture side, advanced neuromorphic processors have been designed to mimic human functions of perception, motor control and multisensory integration.

The submitted manuscripts were reviewed by experts from both academia and industry. After two rounds of reviewing, the highest quality manuscripts were accepted

Chuanchao Huang

carlos_huang@alumni.hust.edu.cn

1 Department of Strategic Planning and Implementation, China Merchants Bank Tower, No. 7088, Shennan Boulevard, Shenzhen 518040, China for this special issue. This special issue will be published by Neural Computing and Applications as special issues. Totally, 19 papers are suggested to EiC for acceptance. The selected papers are summarized as follows.

Zhou et al. [1] propose a novel algorithm named MGGS (modified genetic algorithm (GA) combined with greedy strategy). The proposed algorithm leverages the modified GA algorithm combined with greedy strategy to optimize task scheduling process. Zhang and Zhai [2] propose a new picking algorithm with the emerging new graph signal processing technology in the signal processing field. Financial product price data are treated by $\mathrm{Yu}$ and Yan [3] as a one-dimensional series generated by the projection of a chaotic system composed of multiple factors into the time dimension, and the price series is reconstructed using the time series phase-space reconstruction (PSR) method. In the perspective of government regulation, Liu and $\mathrm{Yu}$ [4] focus on the regulatory warning model of regional product quality. A pseudo-random state transition rule is used by Luo et al. [5] to select path, the state transition probability is calculated according to the current optimal solution and the number of iterations, and the proportion of determined or random selections is adjusted adaptively. Liu and Ran [6] study the selection and evaluation of the partners in the supply chain environment from the point of view of the current research situation of the cooperative relationship in the supply chain environment at home and abroad. Sun et al. [7] mainly address the issue of the power generation fluctuations due to the stochastic characteristics of the renewable energies, which threaten the stability of the power grid. Li and Sun [8] establish a predictive model that combines kernel parameters and parameter optimization to model. The mesh search method, genetic algorithm, and particle swarm optimization algorithm are used to optimize the parameters of the SVM under various kernel functions such as radial basis kernel function. Cui and $\mathrm{Li}$ [9] introduce the basic research results of space fault network. The basic ideas, basic definitions and corresponding physical 
meanings of space fault network are discussed. Xu and Jiang [10] study the current recommendation algorithm, analyze the current research status of the recommendation system, and deeply analyze the research of deep learning in the field of recommendation systems; based on BPSO algorithm, the context complex segmentation method is applied, and then the deep convolutional neural network is applied for feature extraction.

$\mathrm{Ma}$ et al. [11] propose a trusted forensics scheme based on digital image watermark in intelligent VANET, in which they proposed technical and fair algorithms for trusted forensics, and the trusted forensics scheme includes basic forensics parameter data obtaining critical forensics data automatic generation and forensics data extraction. Zhao and Sun [12] develop two profits distribution models under no government subsidies and under government subsidies in closed-loop supply chain. By comparing the members' profits distribution in the two models, they find that government could control the profits distribution pattern by adjusting government subsidy rate. A prototype model named real-time bus routes updated model (RT-BRU) is developed by Zhao et al. [13] to process and mine the GPS trajectory data collected from each urban bus of each interval. Wei et al. [14] study the structure of audio, the analysis and extraction of audio features, the digital audio classifier based on support vector machines (SVM) and the audio segmentation technology based on BCI. In order to increase economic effects of MPD, Zhang and Yuan [15] analyze the MPD system and utilizes wellhead back pressure as the controlled variable. Guo [16] analyzes the current network structure and distribution mode of e-commerce logistics city distribution, and analyzes and discusses the problems existing in current e-commerce logistics city distribution. To bright this gap between the practical requirements and the applications, one convex optimization-based framework with one L0 regularization and the constraint on the learners' characteristics was presented by Lin et al. [17]. Yang et al. [18] apply multiattribute decision making to automobile manufacturing and service industry, taking 14 attributes of manufacturing and six attributes of automobile service as input, and training by BP neural. Zhang et al. [19] establish the correlation model between customer demands and productive service demands based on clustering ideas and mathematical statistics theory to complete the modularization of productive service demands for CNC machine tools.

Acknowledgements The guest editors would like to thank Prof. John MacIntyre who is the editor in chief of Neural Computing and Applications. His help and trust is the most important thing for the success of this SI. The guest editors would like to thank the reviewers for their high-quality reviews, which provided insightful and constructive feedback to the authors of the papers.

\section{References}

1. Zhou Z, Li F, Zhu H et al (2019) An improved genetic algorithm using greedy strategy toward task scheduling optimization in cloud environments. Neural Comput Appl. https://doi.org/10. 1007/s00521-019-04119-7

2. Zhang QJ, Zhai MY (2019) First arrivals picking based on graph signal theory. Neural Comput Appl. https://doi.org/10.1007/ s00521-019-04209-6

3. Yu P, Yan X (2019) Stock price prediction based on deep neural networks. Neural Comput Appl. https://doi.org/10.1007/s00521019-04212-x

4. Liu C, Yu T (2019) The regulatory warning model of regional product quality based on the back-propagation artificial neural network. Neural Comput Appl. https://doi.org/10.1007/s00521019-04188-8

5. Luo Q, Wang H, Zheng Y et al (2019) Research on path planning of mobile robot based on improved ant colony algorithm. Neural Comput Appl. https://doi.org/10.1007/s00521-019-04172-2

6. Liu L, Ran W (2019) Research on supply chain partner selection method based on BP neural network. Neural Comput Appl. https://doi.org/10.1007/s00521-019-04136-6

7. Zhai S, Sun Y, Cui H et al (2019) Adjustable loads control and stochastic stability analysis for multi-energy generation system based on Markov model. Neural Comput Appl. https://doi.org/10. 1007/s00521-019-04120-0

8. Li X, Sun Y (2019) Stock intelligent investment strategy based on support vector machine parameter optimization algorithm. Neural Comput Appl. https://doi.org/10.1007/s00521-019-04566-2

9. Cui T, Li S (2019) Research on basic theory of space fault network and system fault evolution process. Neural Comput Appl. https://doi.org/10.1007/s00521-019-04247-0

10. Xu H, Jiang C (2019) Research on context-aware group recommendation based on deep learning. Neural Comput Appl. https:// doi.org/10.1007/s00521-019-04286-7

11. Ma Z, Jiang M, Huang W (2019) Trusted forensics scheme based on digital watermark algorithm in intelligent VANET. Neural Comput Appl. https://doi.org/10.1007/s00521-019-04246-1

12. Zhao J, Sun N (2019) Government subsidies-based profits distribution pattern analysis in closed-loop supply chain using game theory. Neural Comput Appl. https://doi.org/10.1007/s00521019-04245-2

13. Zhao Y, Liu Y, Ge L et al (2019) Spatiotemporal analysis of trajectory for a new real-time bus routes updated model. Neural Comput Appl. https://doi.org/10.1007/s00521-019-04244-3

14. Wei P, He F, Li L et al (2019) Research on sound classification based on SVM. Neural Comput Appl. https://doi.org/10.1007/ s00521-019-04182-0

15. Zhang H, Yuan X (2019) An improved particle swarm algorithm to optimize PID neural network for pressure control strategy of managed pressure drilling. Neural Comput Appl. https://doi.org/ 10.1007/s00521-019-04192-y

16. Guo K (2019) Research on location selection model of distribution network with constrained line constraints based on genetic algorithm. Neural Comput Appl. https://doi.org/10.1007/s00521019-04257-y

17. Lin J, Li Y, Lian J (2019) A novel recommendation system via L0-regularized convex optimization. Neural Comput Appl. https://doi.org/10.1007/s00521-019-04213-w 
18. Yang M, Zhu H, Guo K (2019) Research on manufacturing service combination optimization based on neural network and multi-attribute decision making. Neural Comput Appl. https://doi. org/10.1007/s00521-019-04241-6

19. Zhang F, Ding H, Zhang N (2019) Productive service demands modularization for $\mathrm{CNC}$ machine tools based on the improved $\mathrm{AP}$ clustering algorithm. Neural Comput Appl. https://doi.org/10. 1007/s00521-019-04173-1

Publisher's Note Springer Nature remains neutral with regard to jurisdictional claims in published maps and institutional affiliations. 